\title{
Dynamics of high risk clinical target volume reduction during brachytherapy and impact on its coverage in patients with inoperable cervical cancer
}

\author{
M. POBIJAKOVA ${ }^{1, *}$, D. SCEPANOVIC ${ }^{1, *}$, M. PALUGA ${ }^{1}$, M. FEKETE ${ }^{1}$, J. MARDIAK $^{2}$ \\ ${ }^{1}$ Radiation Oncology Department, ${ }^{2}$ Clinical Oncology Department, National Cancer Institute of Slovakia, Bratislava, Slovakia \\ *Correspondence: margita.pobijakova@nou.sk; danijela.scepanovic@nou.sk
}

Received January 13, 2017/ Accepted April 10, 2017

\begin{abstract}
Cervical cancer is the third most common cancer in women worldwide. Standard of care for patients with node positive or locally advanced tumors $>4 \mathrm{~cm}$ is definitive radiotherapy and concurrent chemotherapy. Brachytherapy is an integral part of definitive radiotherapy for cervical cancer. The aim of the study was to show a dynamics of High Risk Clinical Target Volume (HR-CTV) reduction during Brachytherapy (BT) as a part of definitive treatment (External Beam Radiotherapy /EBRT/ +/- Chemotherapy /ChT/) depending on the initial Gross Tumor Volume (GTV) and its impact on HR-CTV coverage in patients with inoperable cervical cancer. We analyzed the dosimetric data for BT of 54 patients who have had Three Dimensional Planning of BT (3D BT). The Gross Tumor Volume, HR-CTV and organs at risk (OARs) were contoured on the magnetic resonance imaging (MRI), subsequently on the co-registered MRI images with computed tomography (CT). Point A and ICRU 38 rectal and bladder points were defined on reconstructed CT images. Patients were categorized on the basis of whether the $100 \%$ isodose line of the point-A prescription dose encompassed the HR-CTV (1st group) or not (2nd group). The $30 \mathrm{cc}$ volume has been determined as a cut-off value, which represented the most acceptable value of intermediate size of volumes.

The initial mean value of GTV was $42 \mathrm{cc}$. After completion of EBRT/ChT, the mean GTV was 3.24cc what was $91 \%$ reduction rate in relation to the initial value. We followed the dynamics of HR-CTV reduction during BT and have noted its minimal reduction from $24.3 \mathrm{cc}$ (mean value) at the time of the first fraction to the $24.1 \mathrm{cc}$ before fourth fraction. The mean V100 was $98 \%$ and increased with decreasing of the volume size ( $\mathrm{p}=0.0063$, Fisher's exact test). D90 (mean value was 96.3 Gy10) has been correlated with V100 and also, it increased with decreasing of the volume size ( $\mathrm{p}=0.0003)$. The mean D0.1cc and D2cc of rectum doses were $80 \mathrm{~Gy} 3$ and $65.6 \mathrm{~Gy} 3$, respectively. The mean ICRU rectal dose for all patients was $67.2 \mathrm{~Gy} 3$. The mean D0.1cc (99.5 Gy3), D2cc (79.5 Gy3) and ICRU (75.2 Gy3) of bladder doses were acceptable.

Dynamics of HR-CTV reduction during BT was minimal, although, significant reduction of the GTV was achieved after EBRT/ChT. This study revealed that the dose prescription of $7 \mathrm{~Gy} \times 4$ fractions to point A was not sufficient indicator for dose coverage of the HR-CTV. However, dosimetric parameters as V100 and D90 were strong indicators for coverage of HR-CTV which was inversely related to the volume of the target and the extension of tumor. However, dosimetric parameters for rectum and bladder (D0.1cc, D2cc and ICRU) did not show dependence on the target volumes.
\end{abstract}

Key words: cervical carcinoma, brachytherapy, 3D planning, dosimetric parameters, magnetic resonance imaging, computed tomography

Cervical cancer is the third most common cancer in women worldwide; definitive radiation therapy and concurrent chemotherapy is the accepted standard of care for patients with node positive or locally advanced tumors $>4 \mathrm{~cm}$. Brachytherapy is an important part of definitive radiotherapy [1]. The current treatment of choice includes EBRT with weekly cisplatin followed by intracavitary BT [2]. Tumor shrinkage during whole pelvic radiation allows for a better geometric implant leading to excellent local control and survival in patients with early stage disease. However, for patients with bulky disease or with locally advanced stages, locoregional recurrences remain significant leading to a poor survival [3].

Traditionally, as outlined in the International Commission on Radiation Units and Measurements (ICRU) 38 and the 2000 American Brachytherapy Society (ABS) guidelines for cervical cancer, brachytherapy dose was based on 
two-dimensional (2D) planning prescribed to a modification of the classical Manchester system point A for target coverage and conventional points for critical organs $[4,5]$. However, the doses delivered to tumor and normal tissues from BT are difficult to quantify accurately in conventional BT planning [6].

A modern approach in treatment planning for cervical carcinoma is based on CT sections and on a $3 \mathrm{D}$ dose distribution. This allows better assessment of dose distributions in different volumes, such as GTV, CTV, and OARs (rectum, bladder, and intestines). In 2004, guidelines were published for proposing image-based BT for cervical cancer [7]. Recently, recommendations were given for target and OARs delineation and dose prescription on MRI for image based BT in cervical cancer by the GEC-ESTRO $[8,9]$.

This study was conducted to analyze a dynamics of HR-CTV reduction during BT as a part of definitive treatment (EBRT +/-ChT) depending on the initial GTV and its impact on HR-CTV coverage in patients with inoperable cervical cancer.

\section{Patients and methods}

Between October 2011 and July 2015, 54 patients with biopsy-proven inoperable cervical cancer were enrolled in the prospective study. They underwent radical concurrent chemoradiotherapy consisting of weekly cisplatin plus radiotherapy in the Department of Radiation Oncology at the National Cancer Institute of Slovakia.

All patients were evaluated for staging with routine investigations (blood counts, kidney and liver function test, gynecological examination, CT of thorax and abdomen and diagnostic MRI of the pelvis). The treatment consists of a combination of EBRT with concurrent weekly $40 \mathrm{mg} / \mathrm{m}^{2}$ cisplatin and high dose rate (HDR) intracavitary BT with or without interstitial needles. The most EBRT was planned with a four-field box technique using a treatment planning system (PrecisePlan), though 5 patients had Intensity Modulated Radiotherapy (IMRT). A total of 45-50 Gy dose (1.8-2 Gy/fraction, daily) was delivered using 18-MV photons. Brachytherapy was performed with a remote HDR afterloading technique with Iridium-192 source. The time between the completion of EBRT/ChT and the first BT application was 7 days. The planned dose per fraction was 7 Gy prescribed to point A, given in 4 fractions, and the BT was delivered twice a week.

All patients underwent tandem and ring high-dose rate BT under general anaesthesia, with a CT/MRI-compatible ring applicator (Nucletron Systems, Veenendaal, The Netherlands) having a tandem length of 4 or $6 \mathrm{~cm}$, a curvature of $45^{\circ}$ and a ring diameter of 3 or $3.4 \mathrm{~cm}$ (Vienna applicator). Vagina was packed with a gauze to push away the rectum and bladder and to fix the applicator. Before each application, a urinary catheter was inserted and the catheter balloon inflated with contrast media $(7 \mathrm{~mL})$ to localize the bladder neck. Patients were encouraged to empty their bowels before a procedure and before the next $\mathrm{BT}$ procedures. The probe for in vivo dosimetry has been inserted into the rectum. In addition, eight patients had interstitial needles (range 2-4).

All patients underwent MRI and CT scans followed by contouring on the co-registered images. Before each fraction of BT, all patients had CT but MRI only before the first BT fraction. However, 15 patients had MRI as preplanning. These patients had larger tumor volumes and the preplanning with MRI are used to make decision about addition or not the intersticial needles. MRI was done in the radiodiagnosis department (1.5 Tesla, Siemens). Sagittal, coronal and axial images were obtained, including the tumor, entire cervix, corpus uteri, parametria and vagina. Also, the CT was done in the radiodiagnosis department. No intravenous contrast was used. All MRI and CT slices were transferred via a hospital network to the treatment planning system (Oncentra, Nucletron B.V, The Netherlands).

First, MRI images were obtained followed by the co-registration with $\mathrm{CT}$ and contoured for gross tumor volume (GTV), high-risk clinical target volume (HR-CTV), intermediate-risk clinical target volume (IR-CTV) and organ risks (OARs) on Oncentra brachytherapy planning workstation.

The tumor contouring was based on clinical findings and CT/MRI information. The HR-CTV always included the whole of the cervix as per GEC-ESTRO guidelines, and the parametrial and vaginal extensions were included as per the clinical examination at the time of brachytherapy application. The upper limit of the cervix was defined as the conical two slices above the starting of uterine budge, while the endocervical extensions were contoured as suspected clinically. The IR-CTV was contoured based on the adaptation of GEC-ESTRO recommendations with $1 \mathrm{~cm}$ margin to HR-CTV. The bladder, rectum and sigmoid were contoured as the outer wall of the organ. Applicator reconstruction was done directly on CT with metallic dummy markers being used as a surrogate for source position. Point $\mathrm{A}$ was defined as $2 \mathrm{~cm}$ above the superior surface of the ring and $2 \mathrm{~cm}$ lateral along the uterine axis. The dose received by at least $90 \%$ of the volume (D90) and percentage of volume receiving $100 \%$ or more than the prescribed dose (V100) were calculated using cumulative dose-volume histograms (DVHs) of the CTV (HR-CTV). Also, DVHs were evaluated for the dose to 0.1 and $2 \mathrm{cc}$ for the OARs. The BT dose was added to the EBRT dose to produce the total equivalent dose in $2 \mathrm{~Gy}$ fractions (EQD2) using a $\alpha / \beta$ ratio of $10\left(\mathrm{~Gy}_{10}\right)$ for tumor and $3\left(\mathrm{~Gy}_{3}\right)$ for normal tissues. The dose values for tumor and OARs are reported in the dose/HDR fraction. The 30cc of HR-CTV has been determined as a cut-off value which represented the most acceptable value for intermediate size of volumes.

\section{Results}

The median age of the patients was 51 years (range, 31-78 years). There were $54 \%$ of patients (pts) with Stage T2bN1, $11 \%$ of pts with Stage T2bN0, 11\% of pts with Stage T4N1, $7 \%$ of pts with Stage T3bN1, 6\% of pts with Stage T2bNx, 


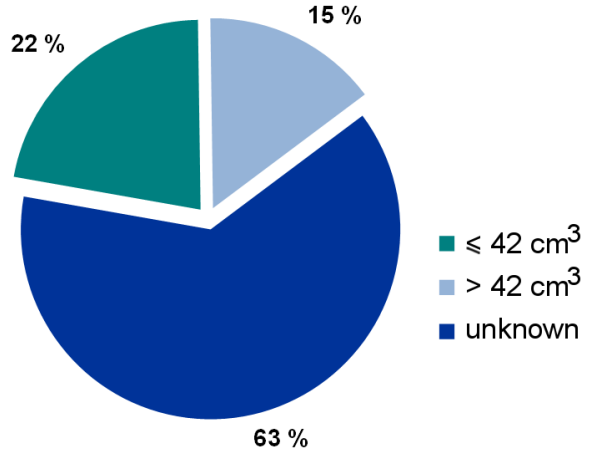

Figure 1. Initial GTVcc (\% of pts)

$4 \%$ of pts with Stage T3aN1, and per 1 patient with Stages T1b1N1, T1b2Nx, T4N0 and T4N2. Patient characteristics are shown in Table 1.

The most patients (63\%) received EBRT to a dose of $45 \mathrm{~Gy}$ in 25 fractions (range 43.2-50.4 Gy) with concurrent cisplatinum-based chemotherapy (mean number of cycles was 5, range 1-6 cycles) followed by four fractions of 7 Gy BT ( 3 patients had $3 \times 7$ Gy). Treatment details are shown in Table 2.

Patients were categorized on the basis of whether the $100 \%$ isodose line of the point-A prescription dose encompassed the HR-CTV (1st group) or not (2nd group). At the time of the first BT fraction, the dose coverage with $100 \%$ at the point A encompassed HR-CTV for 26 patients with a lower volume than $30 \mathrm{cc}$ and 9 patients with volumes larger than $30 \mathrm{cc}$. However, $100 \%$ dose did not encompass HR-CTV for 13 patients with volumes less than $30 \mathrm{cc}$ and for 6 patients with volumes larger than $30 \mathrm{cc}(\mathrm{p}=0.7533$, Fisher's exact test). Also, at the time of the fourth BT fraction, the difference between these two group of pts was not considered to be statistically significant $(\mathrm{p}=0.5490)$.

Before definitive treatment the mean GTV was 42cc which has been measured using a diagnostic MRI. Most patients had initial GTV above 42cc (63\%) (Table 1, Figure 1).

After completion of EBRT/ChT the mean GTV was 3.24cc (range $0.37-20.67 \mathrm{cc}$ ) which has been measured by MRI for planning, and it was $91 \%$ reduction rate in relation to the initial value. The mean GTV before fourth fraction of BT was $2.6 \mathrm{cc}$ (range $0.22-19.93 \mathrm{cc}$ ). We followed dynamics of HR-CTV reduction during BT and a minimal reduction of this volume from mean value of 24.3cc (range 8.9-61.4cc) at the time of first fraction to mean value of $24.1 \mathrm{cc}$ (range 6.9-59.1cc) before fourth fraction has been noted.

The mean V100 was $98 \%$ (range $75.3-100 \%$ ) and increased with decreasing of the volume size ( $\mathrm{p}=0.0063$, Fisher's exact test). D90 (mean value was $96.3 \mathrm{~Gy}_{10} /$ range $71.1-117.3 \mathrm{~Gy}_{10} /$ ) has been correlated with V100 and it also increased with decreasing of the volume size ( $\mathrm{p}=0.0003)$. (Figures 2 and 3 ).

The mean D0.1cc and D2cc of rectum doses were $80 \mathrm{~Gy}_{3}$ (range 56.7-97.8 $\mathrm{Gy}_{3}$ ) and $65.6 \mathrm{~Gy}_{3}$ (range $51.5-73.2 \mathrm{~Gy}_{3}$ ),
Table 1. Patients' characteristics.

\begin{tabular}{lc}
\hline Total number of patients & 54 \\
Age & mean=51 years (range 31-78) \\
Staging & Number of pts \\
T1b1N1 & 1 \\
T1b2Nx & 1 \\
T2bNx & 3 \\
T2bN0 & 6 \\
T2bN1 & 29 \\
T3aN1 & 2 \\
T3bN1 & 4 \\
T4N0 & 1 \\
T4N1 & 6 \\
T4N2 & 1 \\
Histology & \\
Squamous cell carcinoma & 49 \\
Adenocarcinoma & 5 \\
Initial tumor volume (cc) & \\
$\leq 30$ & $8(15 \%)$ \\
$>30$ & $34(63 \%)$ \\
Unkown & $12(22 \%)$ \\
\hline
\end{tabular}

Table 2. Treatment characteristics.

\begin{tabular}{|c|c|}
\hline EBRT techniques & Number of pts \\
\hline Four-field box & 49 \\
\hline IMRT & 5 \\
\hline \multicolumn{2}{|l|}{ Dose } \\
\hline 43.2 Gy (1.8 Gy/fraction daily) & 1 \\
\hline 45 Gy (1.8 Gy/fraction daily) & 34 \\
\hline 46 Gy (2 Gy/fraction daily) & 5 \\
\hline 48.6 Gy (1.8 Gy/fraction daily) & 4 \\
\hline 50.4 Gy (1.8 Gy/fraction daily) & 2 \\
\hline $\begin{array}{l}45-46 \mathrm{~Gy}(1.8-2 \mathrm{~Gy} / \text { fraction daily)+boost } \\
\text { to the residual pelvic lymph nodes with the } \\
\text { range of the dose } 5.4-16 \mathrm{~Gy}\end{array}$ & 8 \\
\hline \multicolumn{2}{|l|}{ Concurrent chemotherapy (weekly cisplatin) } \\
\hline with & $\begin{array}{l}53 \text { (mean number of } \\
\text { cycles }=5 / \text { range } 1-6 / \text { ) }\end{array}$ \\
\hline without & 1 \\
\hline \multicolumn{2}{|l|}{ BT (Ring applicator) } \\
\hline $4 \times 7 \mathrm{~Gy}+{ }^{*}$ intersticial needles $(7 \mathrm{pts})$ & 51 \\
\hline $3 \times 7$ Gy $+{ }^{*}$ intersticial needles (1 patient) & 3 \\
\hline
\end{tabular}

${ }^{\star}$ Intersticial needles (range $2-4$ needles)

respectively. The mean D0.1cc $\left(99.5 \mathrm{~Gy}_{3}\right.$, range $\left.51.9-132.6 \mathrm{~Gy}_{3}\right)$ and D2cc $\left(79.5 \mathrm{~Gy}_{3}\right.$, range $\left.48.6-91.3 \mathrm{~Gy}_{3}\right)$ of bladder doses were acceptable. These DVH parameters were not dependent on the HR-CTV except D0.1cc of rectal doses ( $\mathrm{p}=0.0772$ ), although it has been observed that with increasing of the tumor volumes also DVH parameters increased. (Figures 4 


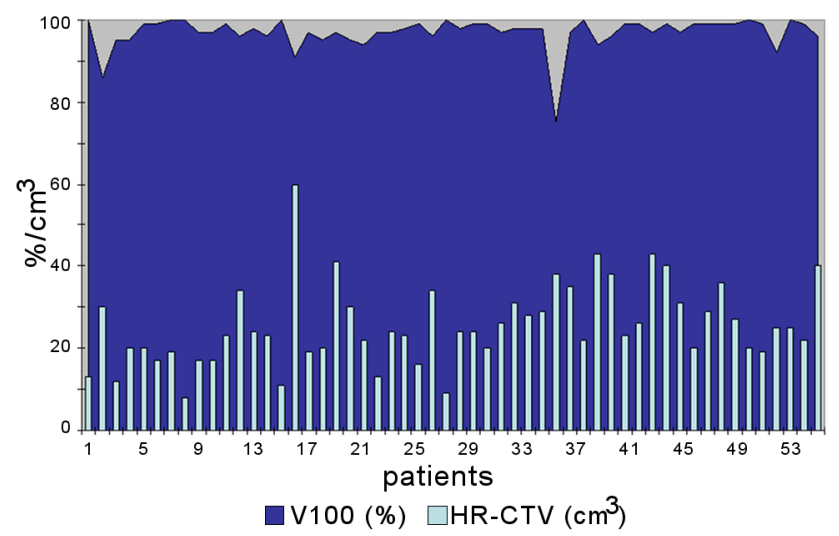

Figure 2. Relationship between V100 and HR-CTV

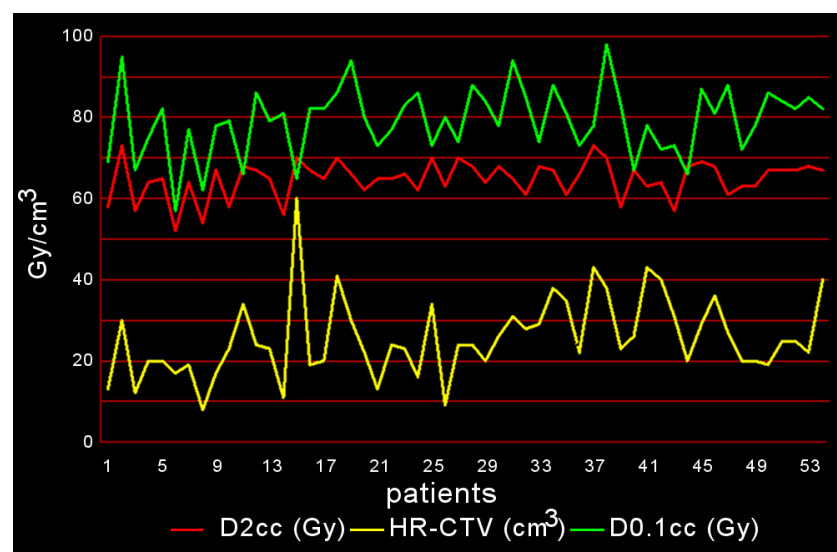

Figure 4. Relationship between D2cc, D0.1cc for rectum and HR-CTV

Table 3. Acute and late toxicity during and after definitive treatment.

\begin{tabular}{lc}
\hline Acute toxicity & Number of pts \\
Without & 40 \\
Gastrointestinal (GI) & 8 \\
Genitourinary (GU) & 1 \\
Unknown & 5 \\
Late toxicity & \\
Without & 41 \\
GI & 5 \\
GU & 3 \\
Combined GI/GU & 1 \\
Unknown & 4 \\
\hline
\end{tabular}

and 5) The mean ICRU rectal dose for all patients was $67.2 \mathrm{~Gy}_{3}\left(55.1-77.6 \mathrm{~Gy}_{3}\right)$ and the mean ICRU bladder dose was $75.2 \mathrm{~Gy}_{3}$ (range 49.9-121.8 $\mathrm{Gy}_{3}$ ).

At the time of first control after completion of the definitive therapy (EBRT $+/-\mathrm{ChT}$ and $\mathrm{BT}), 40$ pts had complete

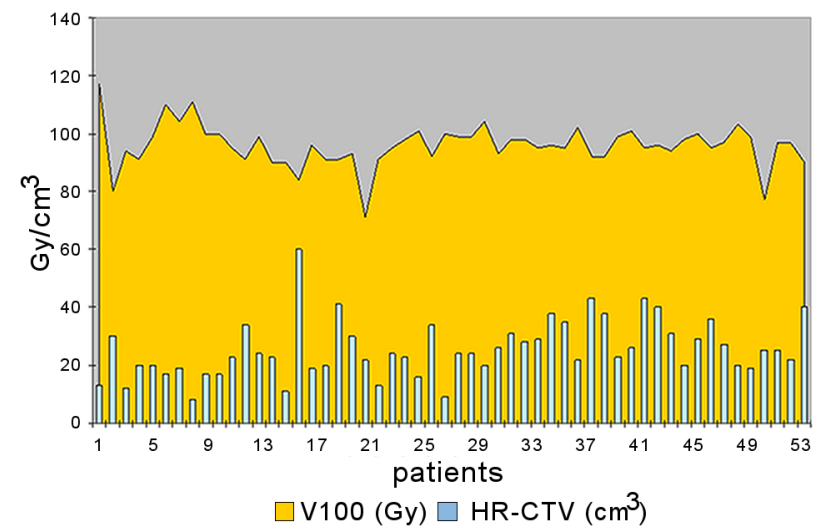

Figure 3. Relationship between D90 and HR-CTV ( $p=0.0003)$

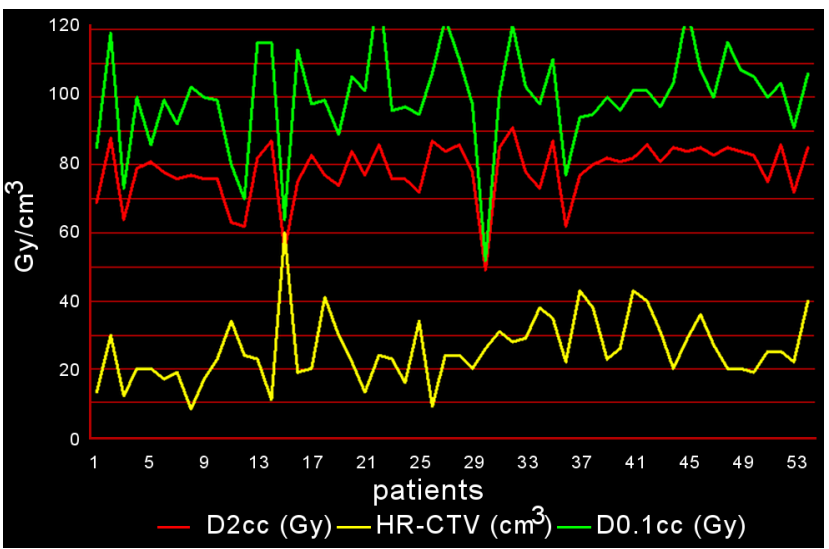

Figure 5. Relationship between D2cc, D0.1cc for bladder and HR-CTV

tumor regression, 9 patients had residual disease, 2 patients local progression of tumor and 3 patients have not been checked. Among patients with residual disease 4 had initial tumor volume larger than $42 \mathrm{cc}, 1$ patient with tumor volume smaller than $42 \mathrm{cc}$ and 4 patients with unknown primary tumor volume. Tumor progression was observed in 1 patient with tumor larger than $42 \mathrm{cc}$ and 1 patient with unknown initial tumor volume. Four patients had distant metastases (retroperitoneal lymph nodes, liver, lung, bones, brain) without findings of local tumor. However, 5 patients had distant metastases (retroperitoneal lymph nodes, lung, bones) also with local progression of tumor. Forty patients (74\%) have had neither no acute toxicity during their definitive treatment nor 41 pts $(76 \%)$ late toxicity. Acute and late toxicity are shown in Table 3.

During the mean time follow-up of 10 months (range 2-43), 5 patients died (1 with local tumor progression, 3 with distant metastases and 1 with second malignant disease /non Hodgkin lymphoma - DLBCL/). Four-year disease-free survival (DFS) was 73\%, and 4-years overall survival (OS) was $92 \%$ in our patients. 


\section{Discussion}

Traditionally, the prescribed dose to point A has been used on the basis of ICRU Report 38 (4). However, this prescribed dose point based on two orthogonal X-ray films was not able to visualize the cervical tumor, and there has been uncertainty about whether the whole cervical tumor is covered with the prescribed dose. Recently, the dose to the HR-CTV has also been used as one of the indices presenting the dose to the cervical tumor $[8,9]$. In our patients, the $100 \%$ isodose line of the point-A prescription dose which sufficiently encompassed or not the HR-CTV did not show dependence on the tumor volumes and there was not a significant difference between these two groups of pts $(p=0.7533$ for 1 st BT fraction and $\mathrm{p}=0.5490$ for 4 th BT fraction).

Cervical tumors rapidly decrease in size during EBRT and BT. The time for $50 \%$ tumor regression has been reported as 21 days, occurring after 30.8 Gy with concurrent chemoradiotherapy $[10,11]$. In our study, the significant regression of GTV has occurred after completion of EBRT/ChT which was $91 \%$. Initially, $63 \%$ of our patients had tumor over $42 \mathrm{cc}$ and we have concluded that there was a significant reduction of such large tumors after definitive therapy. However, GTV decreased slightly during BT for additional 3\%. Depending on the reduction of GTV, the HR-CTV has been decreased and also, we recorded the minimal reduction of HR-CTV during the BT. We assume that the short duration time of $\mathrm{BT}$ was the reason for inexpressive reduction of GTV and consequently HR-CTV.

It is well known that CT alone is inferior to MRI images, and so MRI is taken to be the standard imaging for imagebased contouring for brachytherapy [12]. All of our patients had CT before each fraction of BT and MRI before the first fraction. However, 15 patients with larger tumors (above 42cc) had a MRI as a preplanning which has helped us to decide whether to add the interstitial needles for better coverage of HR-CTV. For large tumors, a combined intracavitary and interstitial technique has been shown to improve tumor coverage in these patients.

Dose volume parameters for target volumes can be derived from cumulative dose volume histogram analysis. Although their clinical relevance has not been proven yet, D100 and D90 are suitable for correct plan comparison of all dose rate techniques. V100 describes how closely the intended treatment could be achieved in terms of target coverage, providing information indirectly on the proportion of the underdosed area. For fractionated treatment, however, D90 and V100 are only usable for evaluation after the last fraction, as it uses summed doses of all fractions [9, 13]. It was reported that 2D BT planning achieved lower HR-CTV and dosages to organs at risk (OARs) in patients with big cervical tumors than in those with small tumors. Harmon et al demonstrated in their study sufficient tumor coverage with lower doses to OARs in HR-CTV $D_{90}$ vs. Point A plans in the small lesion group. These improvements were not seen in the large lesion group, indicating a lesser dosimetric advantage of HR-CTV D90 compared to Point A planning when the cervical lesion is $>25 \mathrm{~cm}^{3}[14,15]$. However, in our study, as expected coverage of the HR-CTV improves when the plan is optimised to a D90 of $100 \%$ of prescription dose rather than prescribing to the A points. The target volume coverage was inversely related to the volume of the target and the extension of tumor.

In fractionated brachytherapy, the location of the high dose region from brachytherapy may not be identical for each fraction. As tumors shrink during the course of radiation, there is a change in tumor volume and configuration over time and consequently, a change in normal tissue topography over time [8]. As organs of interest are hollow, the filling status of the spective organ should be clearly stated, especially for the brachytherapy component. The most constant filling status possible is advised for valid and reliable data collection, especially for the bladder, as this may change within short time periods [9, 16, 17]. Among our patients, dosimetric parameters as D0.1cc, D2cc and ICRU (rectum and bladder) did not indicate dependence on the tumor volume. However, we provided the recommendations for rectal emptiness and constant bladder filling before each fraction which enabled this independence.

Dynamics of HR-CTV reduction during BT was minimal, although, significant reduction of the GTV was achieved after EBRT/ChT. This study revealed that the dose prescription of 7 Gy $\times 4$ fractions to point A was not sufficient indicator for dose coverage of the HR-CTV. However, dosimetric parameters as V100 and D90 were strong indicators for coverage of HR-CTV which was inversely related to the volume of the target and the extension of tumor. However, dosimetric parameters for rectum and bladder (D0.1cc, D2cc and ICRU) did not show dependence on the target volumes.

\section{References}

[1] VARGO JA, BERIWAL S. Image-based brachytherapy for cervical cancer. World J Clin Oncol 2014; 5: 921-930. https:// doi.org/10.5306/wjco.v5.i5.921

[2] VISWANATHAN AN, ERICKSON B, GAFFNEY DK, BERIWAL S, BHATIA SK et al. Comparison and consensus guidelines for delineation of clinical target volume for CTand MR-based brachytherapy in locally advanced cervical cancer. Int J Radiat Oncol Biol Phys 2014; 90(2): 320-328. https://doi.org/10.1016/j.ijrobp.2014.06.005

[3] DUTTA S, NGUYEN NP, VOCK J, KERR C, GODINEZ J et al. Image-guided radiotherapy and brachytherapy for cervical cancer. Front Oncol 2015; 5: 64. https://doi.org/10.3389/ fonc. 2015.00064

[4] International Commission on Radiation Units and Measurements (ICRU). Dose and volume specification for reporting intracavitary therapy in gynecology, ICRU Report, 38. Bethesda, MD: ICRU, 1985 
[5] NAG S, ERICKSON B, THOMADSEN B, ORTON C, DEMANES JD et al. The American Brachytherapy Society recommendations for high-dose-rate brachytherapy for carcinoma of the cervix. Int J Radiat Oncol Biol Phys 2000; 48: 201-211.

[6] ONAL C, ARSLAN G, TOPKAN E, PEHLIVAN B, YAVUZ $\mathrm{M}$ et al. Comparison of conventional and CT-based planning for intracavitary brachytherapy for cervical cancer: target volume coverage and organs at risk doses. J Exp Clin Cancer Res 2009; 28: 95. https://doi.org/10.1186/1756-9966-28-95

[7] NAG S, CARDENES H, CHANG S, DAS IJ, ERICKSON $B$ et al. Proposed guidelines for image-based intracavitary brachytherapy for cervical carcinoma: report from ImageGuided Brachytherapy Working Group. Int J Radiat Oncol Biol Phys 2004; 60: 1160-1172. https://doi.org/10.1016/j. ijrobp.2004.04.032

[8] HAIE-MEDER C, POTTER R, VAN LIMBERGEN E, BRIOT E, DE BRABANDERE $M$ et al. Recommendations from Gynaecological (GYN) GEC-ESTRO Working Group (I): concepts and terms in 3D image based $3 \mathrm{D}$ treatment planning in cervix cancer brachytherapy with emphasis on MRI assessment of GTV and CTV. Radiother Oncol 2005; 74: 235-245. https://doi.org/10.1016/j.radonc.2004.12.015

[9] POTTER R, HAIE-MEDER C, VAN LIMBERGEN E, BARILLOT I, DE BRABANDERE $M$ et al. Recommendations from gynaecological (GYN) GEC ESTRO working group (II): concepts and terms in 3D image-based treatment planning in cervix cancer brachytherapy-3D dose volume parameters and aspects of 3D image-based anatomy, radiation physics, radiobiology. Radiother Oncol 2006; 78: 67-77. https://doi.org/10.1016/j.radonc.2005.11.014

[10] OHKUBO Y, OHNO T, NODA S, KUBO N, NAKAGAWA A et al. Interfractional change of high-risk CTV D90 during image-guided brachytherapy for uterine cervical cancer. J Radiat Res 2013; 54: 1138-1145. https://doi.org/10.1093/jrr/rrt073
[11] LEE CM, SHRIEVE DC, GAFFNEY DK. Rapid involution and mobility of carcinoma of the cervix. Int J Radiat Oncol Biol Phys 2004; 58: 625-630.

[12] ESKANDER RN, SCANDERBEG D, SAENZ CC, BROWN $\mathrm{M}$, YASHAR C. Comparison of computed tomography and magnetic resonance imaging in cervical cancer brachytherapy target and normal tissue contouring. Int J Gynecol Cancer 2010; 20: 47-53. https://doi.org/10.1111/ IGC.0b013e3181c4a627

[13] GOYAL MK, KEHWAR TS, MANJHI J, BARKER JL, HEINTZ BH et al. Dosimetric evaluation of tandem-based cervical high-dose-rate brachytherapy treatment planning using American Brachytherapy Society 2011 recommendations. J Radiother Practice 2016; 15: 283-289. https://doi. org/10.1017/s1460396916000133

[14] HARMON G, DIAK A, SHEA SM, YACOUB JH, SMALL W JR et al. Point A vs. HR-CTV D90 in MRI-based cervical brachytherapy of small and large lesions. Brachytherapy 2016; 15: 825-831. https://doi.org/10.1016/j.brachy.2016.08.010

[15] REN J, YUAN W, WANG R, WANG Q, LI Y et al. Dosimetric Comparison between Three-Dimensional Magnetic Resonance Imaging-Guided and Conventional Two-Dimensional Point A-Based Intracavitary Brachytherapy Planning for Cervical Cancer. PLoS One 2016; 11: e0161932. https:// doi.org/10.1371/journal.pone.0161932

[16] WACHTER-GERSTNER N, WACHTER S, REINSTADLER E, FELLNER C, KNOCKE TH et al. Bladder and rectum dose defined from MRI based treatment planning for cervix cancer brachytherapy: comparison of dose-volume histograms for organ contours and organ wall, comparison with ICRU rectum and bladder reference point. Radiother Oncol 2003; 68: 269-276.

[17] BANERJEE R, KAMRAVA M. Brachytherapy in the treatment of cervical cancer: a review. Int J Womens Health 2014; 6: 555-564. https://doi.org/10.2147/IJWH.S46247 